\title{
Can accident and emergency nurse practitioners interpret radiographs? A multicentre study
}

\author{
S Meek, J Kendall, J Porter, R Freij
}

\begin{abstract}
Objective-To assess the ability of nurse practitioners in accident and emergency (A\&E) to interpret distal limb radiographs, by comparison with senior house officers.

Design-Nurse practitioners and senior house officers in $13 \mathrm{~A} \& \mathrm{E}$ departments or minor injury units were shown 20 radiographs of distal limbs, with brief history and examination findings, and asked to record their interpretation.

Outcome measure-A total score for each subject was calculated by comparing answers against agreed correct responses.

Results-Nurse practitioners in general compared favourably with senior house officers. Those nurse practitioners who interpret radiographs as part of their role in minor injury units performed as well as the experienced senior house officer group.

Conclusions-Nurse practitioners in A\&E are able to interpret radiographs to a standard equal to senior house officers with three to five months' experience. Those nurse practitioners actively interpreting radiographs as part of their role in minor injury units are able to interpret radiographs to the same standard as senior house officers with more than five months' experience.

(F Accid Emerg Med 1998;15:105-107)
\end{abstract}

Keywords: nurse practitioners; senior house officers; $x$ ray interpretation

\section{Accident and}

Emergency

Department, Frenchay

Hospital, Bristol, UK

$\mathrm{J}$ Kendall

Accident and

Emergency

Department, Southend

Hospital,

Southend-on-Sea,

Essex, UK

J Porter

Accident and

Emergency

Department, Central

Middlesex Hospital,

London NW10, UK

R Freij

Correspondence: Steve Meek, Consultant, Accident and Emergency Department, Royal United Hospital, Bath BA1 3NG, UK.

Accepted for publication 24 September 1997 practitioners) tended to order more radiographs than doctors.
Our objectives were to assess the ability of nurse practitioners to identify a range of subtle but clinically important radiographic abnormalities by comparison with $A \& E$ senior house officers and to assess the effect on this skill of training for radiograph interpretation.

\section{Methods}

The study was carried out between January and September 1995. Identical sets of 20 radiographs were shown to nurse practitioners and senior house officers in $13 \mathrm{~A} \& \mathrm{E}$ departments or minor injury units in southern and central England (table 1).

All the radiographs were taken following trauma, and were of the arm distal to or including the elbow, or the leg distal to the knee, as these are the areas nurse practitioners are usually confined to when requesting radiographs. ${ }^{1}$ Although some of the films were of children below the minimum age at which some nurse practitioners can request radiographs, the difficulties in interpreting epiphyseal abnormalities are similar to those in older children. The films were chosen by the first author to represent a wide range of clinically important fractures, dislocations, and common normal variants (table 2). Subjects were told that some of the films were normal.

Agreement on the range of acceptable radiographic diagnoses was obtained by showing the films to three trauma radiologists and three $A \& E$ senior registrars.

The A\&E departments used were chosen originally on the grounds that they employed trained nurse practitioners, all of whom were authorised to request radiographs, and had a consultant or registrar willing to supervise the test. Four further medium sized departments were used to increase the numbers of senior house officers in the study. The three minor injury units used were the only three known to the authors to allow nurse practitioners to interpret radiographs and treat accordingly in the absence of doctors. The nurse practitioners were all trained and officially recognised as such by their departments, though not all had received training in radiograph interpretation.

There is currently no nationally agreed standard training for nurse practitioners.

At Southend, nurse practitioners were authorised to interpret radiographs and treat, but were inclined to limit their role. For the purposes of the study they are considered as trained in radiograph interpretation but not actually undertaking it.

Of 143 nurse practitioners and doctors approached, 142 agreed to take part. We were unable to test every nurse practitioner and 
Table 1 Hospitals involved

Frenchay Hospital, Bristol

Leicester Royal Infirmary

Queen Alexandra, Portsmouth

Lincoln County Hospital

Southend Hospital

St Bartholomew's Hospital

Homerton Hospital

Princess Margaret Hospital, Swindon

Gloucester Royal Hospital

Bristol Royal Infirmary

Southmead Hospital, Bristol

St Charles' Hospital Minor Injuries Unit

St Albans' Hospital Minor Injuries Unit

senior house officer because of annual leave, long term sick leave, and staff turnover; thus $80-100 \%$ of eligible staff were tested in each hospital.

They were provided with a brief history and examination findings for each radiograph: one minute was then allowed for the interpretation of the film, and answers written down, in the presence of an invigilator.

Additional information was sought regarding training and experience. The answer sheets were then scored by two of the authors independently, and the scorers were blind to whether the subject was a nurse practitioner or senior house officer. Subjects were not expected to provide detailed anatomical or orthopaedic knowledge as this would have given the doctors a clear advantage; a clear enough answer to show that the relevant abnormality had been spotted was all that was required. A two mark score was allocated per question to allow 1 to be given for partially correct answers, for example haemarthrosis identified but fracture not seen.

Subjects tested were divided into six groups, defined as follows.

(1) All nurse practitioners-All have received general nurse practitioner training but not necessarily in radiograph interpretation.

(1a) Nurse practitioners: interpreting radiographs - This group all interprets radiographs and treats accordingly. All have had radiograph interpretation training and worked in minor injury units without doctors in the department (though may have visiting clinicians or radiologist).

(1b) Nurse practitioners: radiograph trainedThese were trained to interpret radiographs but did not do so for a variety of

Table 2 The radiographs used

1 Displaced epiphyseal fracture distal radius

2 Angulated epiphyseal fracture distal phalanx of great toe

3 Angulated fracture neck of fifth metacarpal

4 Avulsion fracture of cuboid

5 Lunate dislocation (lateral view)

6 Normal epiphysis fifth metatarsal base

7 Calcaneal fracture (lateral view)

8 Fat pad sign and radial head fracture

9 Glass foreign bodies in two wounds

10 Fat pad sign elbow, no fracture

11 Undisplaced impacted fracture, distal radius

12 Fractured navicular and cuboid on ankle $x$ ray

13 Os trigonum (normal variant)

14 Buckle fracture distal radius

15 Triplanar fracture distal tibia

16 Undisplaced supracondylar fracture and posterior fat pad

17 Fracture base middle phalanx on finger $x$ ray

18 Fracture base fifth metatarsal

19 Fracture medial malleolus

20 Spiral fracture shaft tibia (toddler) reasons, for example because there were experienced doctors in the department or because the hospital had not yet sanctioned this role. Nurses were asked how long their training had been, but the quality of training could not be assessed.

(1c) Nurse practitioners: not radiograph trainedThese fulfilled the definition in (1) but had not had any training in radiograph interpretation.

(2a) Senior house officer, inexperienced-Junior doctors in the first two months of their first $A \& E$ post. Only 23 of 41 (56\%) stated they had received formal training in radiograph interpretation in the last six months, though it is likely many received relevant informal teaching.

(2b) Senior house officer, experienced-These are all in the sixth and final month in A\&E; 28 of $38(74 \%)$ stated they had received formal radiograph interpretation training in the last six months (five did not answer the question).

Statistical analysis was performed by using a single factor analysis of variance between groups, and $95 \%$ confidence intervals calculated using the unpaired $t$ test.

\section{Results}

Fifty eight nurse practitioners, forty three experienced senior house officers, and 41 inexperienced senior house officers were tested, and the mean scores are set out in table 3.

Three nurse practitioners gave no information on training, and are included only in group 1.

The nurse practitioners as a whole (group 1) performed significantly better than the inexperienced senior house officer group (group 2a): the difference between the means was 3.0, 95\% confidence interval 0.3 to 5.7 .

The experienced senior house officer group (group 2b) performed better than the nurse practitioners (group 1) but the difference was not statistically significant: difference between means, $-2.2,95 \%$ confidence interval -4.5 to 0.08 .

The highest mean score was achieved by the nurse practitioners who interpret radiographs at work (group 1a). The difference between this group and the experienced senior house officer group was small and not statistically significant: difference between means, $0.9,95 \%$ confidence interval -3.2 to 5.0 .

The nurse practitioners who interpret radiographs at work (group 1a) also performed better than those trained in radiograph interpretation but not doing so (group 1b): difference between means $4.5,95 \%$ confidence interval 0.35 to 8.65 . There was no significant difference between these groups in duration of radiography training: mean of 5.0 and 6.0 hours respectively (difference between means $1.0,95 \%$ confidence interval -2.5 to 3.5 ).

\section{Discussion}

There is a marked diversity in the role of emergency nurse practitioners. Their remit in the management of minor limb trauma could 
Table 3 Radiograph interpretation scores

\begin{tabular}{llll}
\hline Groups & Number in group & Mean score (max 40) & Confidence interval \\
\hline 1: NP, all & 58 & 22.9 & 21.2 to 24.5 \\
la: NP interpreting & 14 & 26.0 & 21.9 to 30.1 \\
$\quad$ radiographs & & & \\
1b: NP, radiograph trained & 22 & 21.6 & 19.1 to 24.0 \\
1c: NP, not radiograph trained & 19 & 22.8 & 20.5 to 25.2 \\
2a: SHO, inexperienced & 41 & 19.9 & 17.7 to 22.1 \\
2b: SHO, experienced & 43 & 25.1 & 23.4 to 26.7 \\
\hline
\end{tabular}

$\mathrm{NP}$, nurse practitioner; SHO, senior house officer.

include triage, clinical examination, $x$ ray investigation, diagnosis, treatment, and discharge.

It has previously been shown that nurse practitioners can request radiographs effectively, and our study has shown that nurse practitioners can interpret the films as well as senior house officers with three to four months' experience.

Our most important finding is that the nurse practitioners who do interpret radiographs at work are as proficient as the experienced senior house officers. Medical staff rotate to other posts after six months, to be replaced by inexperienced doctors, while senior nurses are more constant: there will always be fluctuations in the standards of the medical staff compared with the less transient senior nurses who continue to gain in competence and confidence.

The quality of radiograph interpretation training is not measurable. However, the mean duration of training was five hours for the nurse practitioners actively practising radiograph interpretation, and six hours for those not actively practising $(p=0.4, N S)$.

Training typically involved small group teaching from radiologists and $\mathrm{A} \& \mathrm{E}$ consultants, with a variable amount of background theory. It seems unlikely that training alone is responsible for the higher scores in the actively interpreting group: it is more likely that training combined with continuous experience and exposure to radiographs leads to the development of this skill.

The performance of nurse practitioners with many years experience but no training in radiograph interpretation provides further evidence for the importance of regular "shop floor" exposure to radiographs: this group had gained interpretation skills without training.

Senior house officers received a variable quantity and quality of radiograph interpretation training, mostly in the first few weeks of the post. In addition to formal tutorials and induction courses, informal shop floor teaching is commonplace. Measuring this training-in terms of both quality and quantity -is difficult and in our study is limited to responses to the question "Have you had formal training/teaching in $x$ ray interpretation in the last six months?" Only $74 \%$ of these experienced senior house officers recalled "formal $x$ ray interpretation" training, and $56 \%$ of the inexperienced group,

The experienced group performed significantly better than the inexperienced group, but it is not possible to identify how much of the improvement was due to training and how much to experience as it was not possible to separate informal training and experience: the answers to the question above suggest that the current quality and quality of formal radiograph interpretation training is not of prime importance-indeed $26 \%$ apparently have not received any such training in the first five months of the post.

The highest mean score for any group was only $26 / 40(65 \%)$, which may appear to be unacceptably low, but the study was designed to focus on the clinically important yet subtle $x$ ray abnormalities, as these are most easily overlooked. We could have used a higher proportion of normal films to simulate real working conditions, and the artificiality of asking candidates to see abnormalities in $18 / 20$ radiographs is a valid criticism of our study. However, despite this we believe the comparison between groups is more important than the actual score: higher scores could have been achieved with easier films, and more realism with more normal films, but the testing would have been less discriminating.

\section{CONCLUSIONS}

Nurse practitioners can interpret distal limb radiographs more accurately than $A \& E$ senior house officers with up to two months' experience, and appear to be equal in this skill to senior house officers with three to five months' experience, even without training. Those nurse practitioners interpreting radiographs at work in minor injury units are as proficient as senior house officers with more than five months' experience.

The expansion of the role of nurse practitioners to include radiograph interpretation appears to be safe, provided the safeguards applied to senior house officer interpretation (radiographer "red dot" system", rapid reporting, and recall) are also used for nurse practitioners. Training for both nurse practitioners and doctors in radiograph interpretation is currently inadequate and both would be expected to perform better with improved training.

Statistical advice was provided by Paul Ewings of Taunton and Somerset NHS Trust. Many thanks to Alain Sauvage, Cliff Mann, Bea Brooks, Paul Younge, and Peter O'Connell who tested many of the subjects. Thank you to all doctors and nurse practitioners who took part.

1 Meek S, Ruffles G, Anderson J, Ohiorenoya D. Nurse practitioners in major accident and emergency departments: titioners in major accident and emergency departments:

2 James MR, Pyrgos N. Nurse practitioners in the accident and emergency department. Arch Emerg Med 1989;6: and emer.

3 Macleod AJ, Freeland P. Should nurses be allowed to request $\mathrm{x}$ rays in an accident and emergency department? Arch Emerg Med 1992;9:19-22.

4 Freij RM, Duffy T, Hackett D, Cunningham D, Fothergill J Radiographic interpretation by nurse practitioners in minor injuries unit. J Accid Emerg Med 1996;13:41-3.

5 Thurston J, Field S. Should accident and emergency nurses request radiographs? Results of a multicentre evaluation. J Accid Emerg Med 1996;13:86-9.

*A "red dot" system is one where the radiographer places a sticker on the film when he or she sees an abnormality, to alert the doctor. 